Bull. Korean Math. Soc. 49 (2012), No. 5, pp. 923-937

http://dx.doi.org/10.4134/BKMS.2012.49.5.923

\title{
DYNAMIC BIFURCATION OF THE PERIODIC SWIFT-HOHENBERG EQUATION
}

\author{
JONGMIN HAN AND MASOUd YARI
}

\begin{abstract}
In this paper we study the dynamic bifurcation of the SwiftHohenberg equation on a periodic cell $\Omega=[-L, L]$. It is shown that the equations bifurcates from the trivial solution to an attractor $\mathcal{A}_{\lambda}$ when the control parameter $\lambda$ crosses the critical value. In the odd periodic case, $\mathcal{A}_{\lambda}$ is homeomorphic to $S^{1}$ and consists of eight singular points and their connecting orbits. In the periodic case, $\mathcal{A}_{\lambda}$ is homeomorphic to $S^{1}$, and contains a torus and two circles which consist of singular points.
\end{abstract}

\section{Introduction}

Fluid motion driven by the thermal gradients is common in nature, especially in geophysical flows such as the atmosphere, the oceans, the mantle of the earth, and the interior of stars. A typical model for fluid convection is the RayleighBénard convection describing a fluid placed between flat horizontal plates such that the lower plate is maintained at a temperature above the upper plate temperature. Due to the thermal expansion, the fluid near the lower plate is less dense and become unstable in the gravitational field. Eventually, we encounter an instability at a finite wave length giving a spatio-temporal pattern formation.

The mathematical model for the Rayleigh-Bénard convection comes from the equation of fluid dynamics in the Boussinesq approximation which involves the Navier-Stokes equations coupled with the temperature equation. In 1977 , Swift and Hohenberg derived in [14] that when the Rayleigh number is near the onset of the convection, the Rayleigh-Bénard convection model may be approximated by the following Swift-Hohenberg equation (SHE)

$$
\frac{\partial u}{\partial t}=-\left(I+\frac{\partial^{2}}{\partial x^{2}}\right)^{2} u+\lambda u-u^{3}, \quad \lambda \in \mathbb{R} .
$$

Received May 3, 2011.

2010 Mathematics Subject Classification. Primary 37G35, 35G25.

Key words and phrases. Swift-Hohenberg equation, attractor bifurcation.

The first author was supported by the Korea Research Foundation Grant funded by the Korean Government (MOEHRD, Basic Research Promotion Fund) (KRF-2008-521-C00020).

The second author was in part supported by a grant from IPM. 
The SHE plays an important role in the studies of pattern formation in many other problems $[1,2]$. It has many qualitatively different stable equilibrium solutions of which are selected as final patterns as time tends to the infinity. In many pattern formation, pattern selections are qualitatively dependent of some control parameters which make bifurcation phenomenon as they passes critical numbers. In this point of view, it is an important subject to study dynamic bifurcation of the SHE describing the instability of the trivial solution and the corresponding pattern forming phenomena.

In this paper, we study how the primary instability occurs according to the control parameter $\lambda$ in the SHE and what is the final patterns. In particular, we consider SHE under the periodic boundary condition

$$
u \text { is periodic on } \Omega=[-L, L] \text {, i.e., } u(-L)=u(L) .
$$

As a simpler case, we also consider the odd-periodic boundary condition

$$
u \text { is periodic on } \Omega=[-L, L] \text { and } u(-x)=-u(x) \text { for all } x \in \Omega \text {. }
$$

To study the dynamic bifurcation of SHE, we use the attractor bifurcation theory developed in $[8,9]$. The main theorem of this theory says that if the trivial solution of governing equation of the system is asymptotically stable at the critical value $\lambda_{0}$ of the control parameter $\lambda$, and if the first eigenvalue of the linearized equation crosses the imaginary axis as $\lambda$ passes $\lambda_{0}$, then the system bifurcates from the trivial solution to an attractor $\mathcal{A}_{\lambda}$. The bifurcated attractor $\mathcal{A}_{\lambda}$ does not contain the trivial solution and thus is different from the global attractor. Moreover, the bifurcated attractor is stable and attracts any bounded set in $H \backslash \Gamma$, where $H$ is the whole phase space and $\Gamma$ is the stable manifold of the trivial solution. This implies that $\mathcal{A}_{\lambda}$ is responsible for the long time dynamics in the stability analysis. From the physical transition point of view, as the control parameter crosses $\lambda_{0}$, the new state after the system undergoes a transition is represented by the whole bifurcated attractor, rather than any of the steady states or any of the connecting orbits [7]. Unlikely to the classical steady state bifurcation theory such as Lyapunov-Schmidt reduction, we study the dynamic system directly in the attractor bifurcation theory and find reduced equations on the center manifold which are essential in the study of phase transitions. The term 'dynamics bifurcation' comes from this reason.

There are several results about the attractor bifurcation for phase transition equations or the pattern formation equations including the complex GinzburgLandau equations [6], the Rayleigh-Bénard convection [10], the Burger's type equation [5], the Kuramoto-Sivashinsky equation (KSE) [8], the Cahn-Hilliard equation (CHE) [11]. Among them the KSE equation and the CHE are fourth order partial differential equations as the SHE. In the point of dynamic bifurcation, the main difference between the SHE and these two fourth order equations is that the eigenvalues of the linearized equation for the SHE have multiplicities while the linearized operator of the KSE and the CHE allow only one sequence of eigenvalues. As we shall see, this makes the final patterns of 
the SHE more fluent than those of the KSE and the CHE. For more discussion, see Remark 2.3 below.

In the next section, we review the known results about the attractor bifurcation of the SHE and make a functional setting. In Section 3, we briefly review of the attractor bifurcation theory in [8] which will be the main tool with the center manifold theory for the proof of main result. In Sections 4 and 5 , we state the main theorems about the dynamic bifurcation of the SHE and provides their proofs.

\section{Preliminaries}

For the functional setting of periodic SHE, let

$$
\begin{aligned}
H & =\left\{u \in L^{2}(\Omega ; \mathbb{R}): u(-L)=u(L) \text { and } \int_{-L}^{L} u(x) d x=0\right\}, \\
H_{p e r}^{4}(\Omega ; \mathbb{R}) & =\left\{u \in H^{4}(\Omega ; \mathbb{R}): \frac{\partial^{j} u}{\partial x^{j}}(-L)=\frac{\partial^{j} u}{\partial x^{j}} u(L) \text { for } j=0,1,2,3\right\}, \\
H_{1} & =H_{p e r}^{4}(\Omega ; \mathbb{R}) \cap H .
\end{aligned}
$$

We also define for odd periodic case

$$
\begin{aligned}
\tilde{H} & =\{u \in H: u(-x)=-u(x), x \in \Omega\}, \\
\tilde{H}_{1} & =H_{\text {per }}^{4}(\Omega ; \mathbb{R}) \cap \tilde{H} .
\end{aligned}
$$

We formulate (1.1) in an abstract equation

$$
\left\{\begin{aligned}
\frac{d u}{d t} & =\mathcal{L}_{\lambda} u+G(u, \lambda), \\
u(0) & =u_{0},
\end{aligned}\right.
$$

by setting $\mathcal{L}_{\lambda} u=-A u+B_{\lambda} u$, and

$$
\begin{aligned}
A u & =\left(\frac{\partial^{2}}{\partial x^{2}}+I\right)^{2}: H_{1} \rightarrow H, \\
B_{\lambda} u & =\lambda I: H_{1} \rightarrow H .
\end{aligned}
$$

We also define nonlinear terms $G(u)=-u^{3}$. It is easy to check that $A, B_{\lambda}, G$ $: H_{1} \rightarrow H\left(\right.$ or $\left.\tilde{H}_{1} \rightarrow \tilde{H}\right)$ are well defined.

Let us investigate the eigenvalues of the operator $\mathcal{L}_{\lambda}$ on $\tilde{H}$. By a simple computation, one can find that $\mathcal{L}_{\lambda}$ has an eigenvalue sequence

$$
\beta_{n}(\lambda)=\lambda-\lambda_{n}, \quad \lambda_{n}=\left[1-\left(\frac{n \pi}{L}\right)^{2}\right]^{2}, \quad n=1,2, \ldots
$$

with the corresponding eigenvectors $\phi_{n}(x)=\sin (n \pi x / L)$. Since $\lambda_{n}$ is a quadratic function of $n \pi / L$, there exists $N \in \mathbb{N}$ such that either

$$
\lambda_{n}>\lambda_{N} \quad \forall n \neq N,
$$

or

$$
\lambda_{n}>\lambda_{N}=\lambda_{N+1} \quad \forall n \neq N, N+1 .
$$


In both cases let

$$
\lambda_{0}(N)=\lambda_{N}=\inf \left\{\lambda_{n}: n \in \mathbb{N}\right\} .
$$

This number plays a crucial role in the dynamic bifurcation in that the primary instability of the system happens at $\lambda_{0}$. Indeed, it was shown in [16] that if $\lambda \leq \lambda_{0}$, then the trivial solution $u=0$ is globally asymptotically stable equilibrium point of (1.1) in $\tilde{H}$ and $H$. As $\lambda$ crosses $\lambda_{0}$, the first eigenvalue $\lambda-\lambda_{0}$ of $\mathcal{L}_{\lambda}$ becomes positive. As a consequence the trivial solution is no longer stable and an attractor bifurcates from the trivial solution.

In order to investigate the bifurcated attractor, we reduce SHE on the center manifolds for each $\lambda$ near $\lambda_{0}$. The dimension of center manifolds is equal to the algebraic multiplicity of the first eigenvalue of $\mathcal{L}_{\lambda}$ which in turn depends on the value of $\lambda_{N}$. Thus, the dimensions of center manifold at $\lambda_{0}$ are different from each other in the cases (2.2) and (2.3) as we shall see. We note that (2.3) occurs when $L=L_{0}(N)$ for some $N \in \mathbb{N}$, where

$$
L_{0}(N)=\left[\frac{\pi^{2}}{2}\left(N^{2}+(N+1)^{2}\right)\right]^{1 / 2} .
$$

Concerning the dynamic bifurcation for SHE in the case (2.2), it was well established in [16] that SHE bifurcate attractors as the control parameter $\lambda$ crosses a critical value $\lambda_{0}$ when $L \leq \pi$. With a slight modification, this result can be extended for the general case (2.2) without assumption that $L \leq \pi$. Here are theorems.

Theorem 2.1. Suppose that (2.2) holds true for some $N \in \mathbb{N}$. Then, for the SHE (1.1) defined in $\tilde{H}$, the primary instability of the system happens at $\lambda_{0}=\lambda_{N}$. Moreover

(a) For $\lambda>\lambda_{0},(1.1)$ bifurcates from $(u, \lambda)=\left(0, \lambda_{0}\right)$ to an attractor $\mathcal{A}_{\lambda}$ which consists of exactly two steady state solutions. For $\lambda$ near $\lambda_{0}$, these solutions can be expressed as

$$
u^{ \pm}(x)= \pm \sqrt{\frac{4\left(\lambda-\lambda_{0}\right)}{3}} \sin \frac{N \pi x}{L}+o\left(\sqrt{\lambda-\lambda_{0}}\right) .
$$

(b) For any bounded open set $U \subset \tilde{H}$ with $0 \in U$, there exists $\varepsilon>0$ such that as $\lambda_{0}<\lambda<\lambda_{0}+\varepsilon, \mathcal{A}_{\lambda}$ attracts $U \backslash \Gamma$ in $\tilde{H}$, where $\Gamma$ is the stable manifold of $u=0$ with codimension 1 .

Theorem 2.2. Suppose that (2.2) holds true for some $N \in \mathbb{N}$. Then, for the SHE (1.1) defined in $H$, the primary instability of the system happens at $\lambda_{0}=\lambda_{N}$. Moreover,

(a) For $\lambda>\lambda_{0}$, (1.1) bifurcates from $(u, \lambda)=\left(0, \lambda_{0}\right)$ to an attractor $\mathcal{A}_{\lambda}$ which is homeomorphic to $S^{1}$. Moreover, $\mathcal{A}_{\lambda}$ consists of steady state solutions and is given by

$$
\left\{y_{1} \sin \frac{N \pi}{L} x+y_{2} \cos \frac{N \pi}{L} x+o(|y|): y_{1}^{2}+y_{2}^{2}=\frac{4}{3}\left(\lambda-\lambda_{0}\right)\right\} .
$$


(b) For any bounded open set $U \subset \tilde{H}$ with $0 \in U$, there exists $\varepsilon>0$ such that as $\lambda_{0}<\lambda<\lambda_{0}+\varepsilon, \mathcal{A}_{\lambda}$ attracts $U \backslash \Gamma$ in $H$, where $\Gamma$ is the stable manifold of $u=0$ with codimension 2 .

If $L \leq \pi$, then it is easy to see that the case (2.4) does not occur and hence (2.3) does not hold true. The purpose of this paper is to study the dynamic bifurcation of SHE under the condition (2.3). This improves the result of [16]. In this case, algebraic multiplicity of $\lambda_{0}$ is twice of the multiplicity in the case (2.3). In the next section, we briefly review of the attraction bifurcation theory developed in [8]. Using this theory, in Sections 4 and 5, we establish the attractor bifurcation of the SHE for the case (2.3) with the odd-periodic and the periodic boundary conditions.

Remark 2.3. The fourth order equations play important roles in the study of pattern formation. Among them, the Kuramoto-Sivashinsky equation (KSE) describes the fluctuations of the position of a flame front, the motion of a fluid going down a vertical wall, or a spatially uniform oscillating chemical reaction in a homogeneous medium [15]. The equation is written as

$$
u_{t}+u_{x x x x}+\lambda u_{x x}+u u_{x}=0
$$

which has the form (3.1) with $\mathcal{L}_{\lambda} u=-A u+B_{\lambda} u$ and

$$
A u=-u_{x x x x}, \quad B_{\lambda} u=-\lambda u_{x x}, \quad G(u)=-u u_{x} .
$$

The eigenvalues of $\mathcal{L}_{\lambda}$ are

$$
\alpha_{n}(\lambda)=\left(\frac{n \pi}{L}\right)^{2}\left\{\lambda-\left(\frac{n \pi}{L}\right)^{2}\right\}=: \tau_{n}\left(\lambda-\tau_{n}\right) .
$$

Thus, the primary instability arises when $\lambda$ crosses $\tau_{1}=\pi^{2} / L^{2}$ and we cannot expect multiplicity like (2.3). Even when we consider the case (2.2) for SHE, the primary instability can arise at $\lambda_{1}$ as well as at any number $\lambda_{N}$ according to the periodic length $L$. Thus SHE allows more phase transition phenomena than KSE as discussed in the introduction. This observation of the dependence of the bifurcation about SHE on $L$ have been made by many authors. For instance, see $[3,12,13]$. One can refer to Chapter 9 of [8] for the dynamic bifurcation for KSE.

\section{Review of the attractor bifurcation theory}

In this section, we briefly review the attractor bifurcation theory developed by Ma and Wang in [8], which is essential to state the main result of this paper.

Let $H_{1}$ and $H$ be two Hilbert spaces with a dense inclusion $H_{1} \hookrightarrow H$. Let us consider the nonlinear evolution equation

$$
\left\{\begin{aligned}
\frac{d u}{d t} & =L_{\lambda} u+G(u, \lambda), \\
u(0) & =u_{0},
\end{aligned}\right.
$$


where $u:[0, \infty) \rightarrow H$ is the unknown function and $\lambda \in \mathbb{R}$ is the system parameter. The parameterized operator $L_{\lambda}: H_{1} \rightarrow H$ are linear completely continuous fields depending continuously on $\lambda$ and satisfy

$$
\begin{cases}L_{\lambda}=-A+B_{\lambda}, & \text { a sectorial operator, } \\ A: H_{1} \rightarrow H, & \text { a linear homeomorphism, } \\ B_{\lambda}: H_{1} \rightarrow H, & \text { a parameterized linear compact operator. }\end{cases}
$$

Then, $L_{\lambda}$ generates an analytic semigroup $\left\{S_{\lambda}(t)=e^{-t L_{\lambda}}\right\}_{t \geq 0}$ and we can define fractional power operators $L_{\lambda}^{\alpha}$ for any $0 \leq \alpha \leq 1$ with domain $H_{\alpha}=$ $\mathcal{D}\left(L_{\lambda}^{\alpha}\right)$. Moreover, $H_{0}=H$ and if $\alpha_{1}>\alpha_{2}$, then $H_{\alpha_{1}} \subset H_{\alpha_{2}}$. We assume that $G(\cdot, \lambda): H_{\alpha} \rightarrow H$ are parameterized $C^{r}$ bounded operators for some $0 \leq \alpha<1$ and $r \geq 0$, and depend continuously on $\lambda$ such that

$$
G(u, \lambda)=o\left(\|u\|_{H_{\alpha}}\right), \quad \forall \lambda \in \mathbb{R} .
$$

In this paper, we are interested in the case that there exists an eigenvalue sequence $\left\{\rho_{k}\right\} \subset \mathbb{C}$ and eigenvector sequence $\left\{e_{k}, h_{k}\right\} \subset H_{1}$ of $A$ satisfying

$$
\left\{\begin{array}{l}
A z_{k}=\rho_{k} z_{k}, \quad z_{k}=e_{k}+i h_{k}, \\
\left\{e_{k}, h_{k}\right\} \text { is a basis of } H, \\
\operatorname{Re} \rho_{k} \rightarrow \infty \text { as } k \rightarrow \infty, \\
\left|\frac{\operatorname{Im} \rho_{k}}{b+\operatorname{Re} \rho_{k}}\right| \leq C
\end{array}\right.
$$

for some constants $b, C>0$. The condition (3.4) implies that $A$ is a sectorial operator. Hence, we can define fractional power operators $A^{\alpha}$ with domain $H_{\alpha}=\mathcal{D}\left(A^{\alpha}\right)$ for any $0 \leq \alpha \leq 1$. For the compact operator $B_{\lambda}: H_{1} \rightarrow H$, we assume that there exists a constant $0 \leq \theta<1$ such that

$$
B_{\lambda}: H_{\theta} \rightarrow H \text { is bounded for all } \lambda \in \mathbb{R} .
$$

It is known that $L_{\lambda}=-A+B_{\lambda}$ is a sectorial operator if (3.4) and (3.5) hold. In this case, $\mathcal{D}\left(L^{\alpha}\right)=\mathcal{D}\left(A^{\alpha}\right)$.

Let $\beta_{1}(\lambda), \ldots, \beta_{k}(\lambda), \ldots \in \mathbb{C}$ be the eigenvalues of $L_{\lambda}$ counting multiplicities. Suppose that

$$
\operatorname{Re} \beta_{j}(\lambda)=\left\{\begin{array}{ll}
<0, & \text { if } \lambda<\lambda_{0} \\
=0, & \text { if } \lambda=\lambda_{0} \\
>0, & \text { if } \lambda>\lambda_{0}
\end{array} \quad(1 \leq j \leq m)\right.
$$

and

$$
\operatorname{Re} \beta_{j}\left(\lambda_{0}\right)<0, \quad \forall j \geq m+1 .
$$

We define the eigenspace of $L_{\lambda}$ at $\lambda_{0}$ by

$$
E_{0}=\bigcup_{j=1}^{m} \bigcup_{k=1}^{\infty}\left\{u \in H_{1}:\left(L_{\lambda_{0}}-\beta_{j}\left(\lambda_{0}\right)\right)^{k} u=0\right\} .
$$

Then, it is known that $\operatorname{dim} E_{0}=m$. 
DYNAMIC BIFURCATION OF THE PERIODIC SWIFT-HOHENBERG EQUATION 929

In order to state the attractor bifurcation theory we need the following definition.

Definition. (i) A set $\Sigma \subset H$ is a (positive) invariant set of (3.1) if $S_{\lambda}(t) \Sigma=\Sigma$ for any $t \geq 0$.

(ii) An invariant set $\Sigma \subset H$ of (3.1) is an attractor if $\Sigma$ is compact and there exists a neighborhood $U \subset H$ of $\Sigma$ such that

$$
\lim _{t \rightarrow \infty} \operatorname{dist}_{H}\left(u_{\lambda}\left(t ; u_{0}\right), \Sigma\right)=0
$$

for any $u_{0} \in U$. The largest open set $U$ satisfying the above condition is the basin of attraction of $\Sigma$.

Definition. (i) We say that (3.1) bifurcates from $(u, \lambda)=\left(0, \lambda_{0}\right)$ to an invariant set $\Omega_{\lambda}$ if there exists a sequence of invariant sets $\left\{\Omega_{\lambda_{n}}\right\}$ of $(3.1)$ with $0 \notin \Omega_{\lambda_{n}}$ such that

$$
\lim _{n \rightarrow \infty} \max _{u \in \Omega_{\lambda_{n}}}|u|=0, \quad \lim _{n \rightarrow \infty} \lambda_{n}=\lambda_{0}
$$

(ii) If the invariant sets $\Omega_{\lambda}$ are attractors of (3.1), then the bifurcation is called an attractor bifurcation.

(iii) If the invariant sets $\Omega_{\lambda}$ are attractors and are homotopy equivalent to an $m$-dimensional sphere $S^{m}$, then the bifurcation is called an $S^{m}$-attractor bifurcation.

The following dynamic bifurcation theorem for (3.1), which comes from Theorem 6.1 of [8], is the main tool for the study of SHE in this paper.

Theorem 3.1 (Attractor Bifurcation Theorem). Suppose that (3.2)-(3.7) hold true, and $u=0$ is a locally asymptotically stable equilibrium point of (3.1) at $\lambda=\lambda_{0}$. Then, we have the following:

(a) The equation (3.1) bifurcates from $(u, \lambda)=\left(0, \lambda_{0}\right)$ to an attractor $\mathcal{A}_{\lambda}$ for $\lambda>\lambda_{0}$, with $m-1 \leq \operatorname{dim} \mathcal{A}_{\lambda} \leq m$, which is connected if $m>1$.

(b) For any $u_{\lambda} \in \mathcal{A}_{\lambda}, u_{\lambda}$ can be expressed as

$$
u_{\lambda}=v_{\lambda}+o\left(\left\|v_{\lambda}\right\|_{H_{1}}\right), \quad v_{\lambda} \in E_{0} .
$$

(c) If $u=0$ is globally asymptotically stable for (3.1) at $\lambda=\lambda_{0}$, then for any bounded open set $U \subset H$ with $0 \in U$, there exists $\varepsilon>0$ such that as $\lambda_{0}<\lambda<\lambda_{0}+\varepsilon, \mathcal{A}_{\lambda}$ attracts $U \backslash \Gamma$ in $H$, where $\Gamma$ is the stable manifold of $u=0$ with codimension $m$. In particular, if (3.1) has a global attractor for all $\lambda$ near $\lambda_{0}$, then $\varepsilon$ can be chosen independent of $U$.

To investigate the stability and bifurcation of (3.1), it is crucial to reduce it to the center manifold. Suppose that $H_{1}$ and $H$ are decomposed into

$$
\left\{\begin{array}{l}
H_{1}=E_{1}^{\lambda} \oplus E_{2}^{\lambda}, \quad H=\tilde{E}_{1}^{\lambda} \oplus \tilde{E}_{2}^{\lambda}, \\
E_{1}^{\lambda} \text { and } E_{2}^{\lambda} \text { are invariant subspaces of } L_{\lambda}, \\
\operatorname{dim} E_{1}^{\lambda}<\infty, \quad E_{1}^{\lambda}=\tilde{E}_{1}^{\lambda}, \\
\tilde{E}_{2}^{\lambda} \text { is the closure of } E_{2}^{\lambda} \text { in } H,
\end{array}\right.
$$


for $\lambda$ near $\lambda_{0}$. In addition, $L_{\lambda}$ can be decomposed into $\mathcal{L}_{\lambda}=\mathcal{L}_{1}^{\lambda} \oplus \mathcal{L}_{2}^{\lambda}$ such that for $\lambda$ near $\lambda_{0}$,

$$
\mathcal{L}_{1}^{\lambda}=\left.\mathcal{L}_{\lambda}\right|_{E_{1}^{\lambda}}: E_{1}^{\lambda} \rightarrow \tilde{E}_{1}^{\lambda}, \quad \mathcal{L}_{2}^{\lambda}=\left.\mathcal{L}_{\lambda}\right|_{E_{2}^{\lambda}}: E_{2}^{\lambda} \rightarrow \tilde{E}_{2}^{\lambda}
$$

where the eigenvalues of $\mathcal{L}_{2}^{\lambda}$ possess negative real part and the eigenvalues of $\mathcal{L}_{1}^{\lambda}$ possess nonnegative real parts at $\lambda=\lambda_{0}$. We can rewrite (3.1) as

$$
\left\{\begin{aligned}
\frac{d v}{d t} & =\mathcal{L}_{1}^{\lambda} v+P_{1} G(v+w, \lambda), \\
\frac{d w}{d t} & =\mathcal{L}_{1}^{\lambda} w+P_{2} G(v+w, \lambda),
\end{aligned}\right.
$$

where $u=v+w \in H_{1}, v \in E_{1}^{\lambda}, w \in E_{2}^{\lambda}$, and $P_{j}: H \rightarrow \tilde{E}_{j}^{\lambda}$ are canonical projections. We have the following Center Manifold Theorem [4].

Theorem 3.2. Assume (3.2), (3.3), (3.8) and (3.9). Let $E_{2}^{\lambda}(\alpha)$ be the closure of $E_{2}^{\lambda}$ in $H_{\alpha}$, where $\alpha \in[0,1)$ is given by (3.3). Then, there exist a neighborhood of $\lambda_{0}$ given by $\left|\lambda-\lambda_{0}\right|<\delta$ for some $\delta>0$, a neighborhood $\mathcal{O}_{\lambda} \subset E_{1}^{\lambda}$ of $v=0$, and a $C^{1}$ function $\Phi(\cdot, \lambda): \mathcal{O}_{\lambda} \rightarrow E_{2}^{\lambda}(\alpha)$ such that the following hold:

(a) $\Phi(0, \lambda)=0$ and $D_{v} \Phi(0, \lambda)=0$.

(b) The set

$$
M_{\lambda}=\left\{(u, v) \in H_{1} \mid v \in \mathcal{O}_{\lambda}, w=\Phi(v, \lambda) \in E_{2}^{\lambda}(\alpha)\right\},
$$

called the center manifold, are locally invariant for $(3.1)$, i.e., if $u_{\lambda}\left(t, u_{0}\right)$ is the solution of (3.1) for $u_{0} \in M_{\lambda}$, then there exists $t\left(u_{0}\right)>0$ such that $u_{\lambda}\left(t, u_{0}\right) \in$ $M_{\lambda}$ for all $0 \leq t<t\left(u_{0}\right)$.

(c) If $\left(v_{\lambda}(t), w_{\lambda}(t)\right)$ is a solution of (3.10), then there are $c_{\lambda}>0$ and $K_{\lambda}>0$ depending $\left(v_{\lambda}(0), w_{\lambda}(0)\right)$ such that

$$
\left\|w_{\lambda}(t)-\Phi\left(v_{\lambda}(t), \lambda\right)\right\|_{H} \leq K_{\lambda} e^{-c_{\lambda} t}
$$

By the Center Manifold Theorem, the equation (3.10) is reduced to

$$
\frac{d v}{d t}=\mathcal{L}_{1}^{\lambda} v+P_{1} G(v+\Phi(v, \lambda), \lambda)
$$

in an neighborhood of 0 in $E_{1}^{\lambda}$.

Finally, we introduce a useful theorem when we study the structure of the bifurcated attractor of (3.11) for the two dimensional case. We consider a two dimensional system

$$
\frac{d \mathbf{y}}{d t}=\beta(\lambda) \mathbf{y}-G(\mathbf{y}, \lambda), \quad \mathbf{y} \in \mathbb{R}^{n} .
$$

Here, $\beta(\lambda)$ is a continuous function of $\lambda$ and $G(\mathbf{y}, \lambda)=G_{k}(\mathbf{y}, \lambda)+o\left(|\mathbf{y}|^{k}\right)$ is a two dimensional vector field for some integer $k=2 m+1 \geq 3$, where $G_{k}\left(\mathbf{y}_{1}, \ldots, \mathbf{y}_{k}, \lambda\right)$ is a $k$-multiple linear function and $G_{k}(\mathbf{y}, \lambda)=G_{k}(\mathbf{y}, \ldots, \mathbf{y}$, $\lambda)$. The following theorem from $[8,9]$ gives a criterion when the system (3.12) bifurcates to an $S^{n-1}$-attractor. 
DYNAMIC BIFURCATION OF THE PERIODIC SWIFT-HOHENBERG EQUATION 931

Theorem 3.3. Suppose that

$$
\beta(\lambda) \begin{cases}<0 & \text { if } \lambda<\lambda_{0}, \\ =0 & \text { if } \lambda=\lambda_{0}, \\ >0 & \text { if } \lambda>\lambda_{0},\end{cases}
$$

and $G_{k}(\mathbf{y}, \lambda)$ satisfies

$$
C_{1}|\mathbf{y}|^{k+1} \leq<G_{k}(\mathbf{y}, \lambda), \mathbf{y}>_{\mathbb{R}^{n}} \leq C_{2}|\mathbf{y}|^{k+1}
$$

for some constants $C_{1}, C_{2}>0$. Then, for $\lambda>\lambda_{0}$, the system (3.12) bifurcates from $(\mathbf{y}, \lambda)=\left(0, \lambda_{0}\right)$ to an attractor $\mathcal{A}_{\lambda}$ which is homeomorphic to $S^{n-1}$.

\section{Odd periodic case}

The first main result is the following involving the the attractor bifurcation of the SHE for the case (2.3) under the odd-periodic boundary condition.

Theorem 4.1. Suppose that (2.3) holds true for some $N \in \mathbb{N}$. Then, for the SHE (1.1) defined in $\tilde{H}$, the primary instability of the system happens at $\lambda_{0}=\lambda_{N}$. Moreover,

(a) If $\lambda \leq \lambda_{0}$, then $u=0$ is globally asymptotically stable equilibrium point of (1.1).

(b) For $\lambda>\lambda_{0}$, (1.1) bifurcates from $(u, \lambda)=\left(0, \lambda_{0}\right)$ to an attractor $\mathcal{A}_{\lambda}$ with $\operatorname{dim} \mathcal{A}_{\lambda}=1$.

(c) For any bounded open set $U \subset \tilde{H}$ with $0 \in U$, there exists $\varepsilon>0$ such that as $\lambda_{0}<\lambda<\lambda_{0}+\varepsilon, \mathcal{A}_{\lambda}$ attracts $U \backslash \Gamma$ in $\tilde{H}$, where $\Gamma$ is the stable manifold of $u=0$ with codimension 2 .

(d) The bifurcated attractor $\mathcal{A}_{\lambda}$ is homeomorphic to $S^{1}$ and consists of eight singular points and their connecting orbits. For $\lambda$ near $\lambda_{0}$, the singular points can be expressed as

$$
\begin{aligned}
& u_{1}^{ \pm}(x)= \pm \frac{2}{3} \sqrt{\lambda-\lambda_{0}}\left\{\sin \frac{N \pi x}{L}+\sin \frac{(N+1) \pi x}{L}\right\}+o\left(\sqrt{\lambda-\lambda_{0}}\right), \\
& u_{2}^{ \pm}(x)= \pm \frac{2}{3} \sqrt{\lambda-\lambda_{0}}\left\{\sin \frac{N \pi x}{L}-\sin \frac{(N+1) \pi x}{L}\right\}+o\left(\sqrt{\lambda-\lambda_{0}}\right), \\
& u_{3}^{ \pm}(x)= \pm \sqrt{\frac{4\left(\lambda-\lambda_{0}\right)}{3}} \sin \frac{N \pi x}{L}+o\left(\sqrt{\lambda-\lambda_{0}}\right), \\
& u_{4}^{ \pm}(x)= \pm \sqrt{\frac{4\left(\lambda-\lambda_{0}\right)}{3}} \sin \frac{(N+1) \pi x}{L}+o\left(\sqrt{\lambda-\lambda_{0}}\right) .
\end{aligned}
$$

Moreover, on the center manifold $u_{1}^{ \pm}, u_{2}^{ \pm}$are saddle points and $u_{3}^{ \pm}, u_{4}^{ \pm}$are stable nodes. 
Proof. For the existence of bifurcated attractor, we verify each condition in Theorem 3.1. From the setting in Section 2, it is trivial to check that (3.2)(3.5) hold true. We note that

$$
\beta_{N}(\lambda)=\beta_{N+1}(\lambda)= \begin{cases}<0, & \text { if } \lambda<\lambda_{0} \\ =0, & \text { if } \lambda=\lambda_{0} \\ >0, & \text { if } \lambda>\lambda_{0}\end{cases}
$$

and $\operatorname{Re} \beta_{n}\left(\lambda_{0}\right)<0$ for all $n \neq N, N+1$. Hence, (3.6)-(3.7) are valid. Moreover, $u=0$ is shown to be globally asymptotically stable for $\lambda \leq \lambda_{0}$ as proved in [16]. Now, we can apply the attractor bifurcation Theorem 3.1 to obtain a bifurcated attractor satisfying the assertions (a), (b), and (c).

It remains to prove the statement (d). First we show that $\mathcal{A}_{\lambda}$ is homeomorphic to $S^{1}$. Let $E_{1}=\operatorname{span}\left\{\phi_{N}, \phi_{N+1}\right\}$ and $E_{2}=E_{1}^{\perp}$ in $\tilde{H}$. Let $P_{j}: \tilde{H} \rightarrow E_{j}$ be the canonical projections and $\mathcal{L}_{j}^{\lambda}=\left.\mathcal{L}_{\lambda}\right|_{E_{j}}$ for $j=1,2$. For $u \in \tilde{H}$, we can write $u=\sum_{n=1}^{\infty} y_{n} \phi_{n}$. If $\Phi(\cdot, \lambda): E_{1} \rightarrow E_{2}$ is a center manifold function and $v=P_{1} u$, then the reduced equation of (1.1) on the center manifold is

$$
\frac{d v}{d t}=\mathcal{L}_{1}^{\lambda} v+P_{1} G\left(y_{N} \varphi_{N}+y_{N+1} \varphi_{N+1}+\Phi\left(y_{N}, y_{N+1}, \lambda\right)\right)
$$

which is equivalent to

(4.15)

$$
\left\{\begin{array}{c}
\frac{d y_{N}}{d t}=\beta_{N} y_{N}+\frac{1}{L}\left\langle G\left(y_{N} \phi_{N}+y_{N+1} \phi_{N+1}+\Phi\left(y_{N}, y_{N+1}, \lambda\right)\right), \phi_{N}\right\rangle, \\
\frac{d y_{N+1}}{d t}=\beta_{N+1} y_{N+1}+\frac{1}{L}\left\langle G\left(y_{N} \phi_{N}+y_{N+1} \phi_{N+1}+\Phi\left(y_{N}, y_{N+1}, \lambda\right)\right), \phi_{N+1}\right\rangle .
\end{array}\right.
$$

Since $\Phi\left(y_{N}, y_{N+1}, \lambda\right)=o(|\mathbf{y}|)$ with $\mathbf{y}=\left(y_{N}, y_{N+1}\right)$ and $G(u)=-u^{3}$, we obtain

$$
\begin{gathered}
\left\langle G\left(y_{N} \phi_{N}+y_{N+1} \phi_{N+1}+\Phi\left(y_{N}, y_{N+1}, \lambda\right)\right), \phi_{N}\right\rangle \\
=-\int_{-L}^{L}\left(y_{N}^{3} \phi_{N}^{4}+3 y_{N}^{2} y_{N+1} \phi_{N}^{3} \phi_{N+1}+3 y_{N} y_{N+1}^{2} \phi_{N}^{2} \phi_{N+1}^{2}\right. \\
\left.+y_{N+1}^{3} \phi_{N} \phi_{N+1}^{3}\right) d x+o\left(|\mathbf{y}|^{3}\right) \\
=-\frac{3 L}{4}\left(y_{N}^{3}+2 y_{N} y_{N+1}^{2}\right)+o\left(|\mathbf{y}|^{3}\right) .
\end{gathered}
$$

A similar computation yields

$$
\begin{aligned}
& \left\langle G\left(y_{N} \phi_{N}+y_{N+1} \phi_{N+1}+\Phi\left(y_{N}, y_{N+1}, \lambda\right)\right), \phi_{N+1}\right\rangle \\
= & -\frac{3 L}{4}\left(2 y_{N}^{2} y_{N+1}+y_{N+1}^{3}\right)+o\left(|\mathbf{y}|^{3}\right) .
\end{aligned}
$$

Hence, the bifurcation equation (4.15) becomes

$$
\frac{d \mathbf{y}}{d t}=\beta(\lambda) \mathbf{y}-F(\mathbf{y})+o\left(|\mathbf{y}|^{3}\right)
$$


DYNAMIC BIFURCATION OF THE PERIODIC SWIFT-HOHENBERG EQUATION 933 where $\beta(\lambda)=\beta_{N}(\lambda)$ and

$$
F(\mathbf{y})=\frac{3}{4}\left(y_{N}^{3}+2 y_{N} y_{N+1}^{2}, 2 y_{N}^{2} y_{N+1}+y_{N+1}^{3}\right) .
$$

It is easy to see that

$$
\frac{3}{4}|\mathbf{y}|^{4} \leq\langle F(\mathbf{y}), \mathbf{y}\rangle_{\mathbb{R}^{2}} \leq \frac{3}{2}|\mathbf{y}|^{4} .
$$

Hence, we can use Theorem 3.3 to show that the bifurcated attractor $\mathcal{A}_{\lambda}$ is homeomorphic to $S^{1}$.

Finally, we show that $\mathcal{A}_{\lambda}$ consists of four minimal attractor, four saddle points, and their connecting orbits. It is known that under nondegenerate conditions the bifurcated equation (4.16) and its truncation

$$
\frac{d \mathbf{y}}{d t}=\beta(\lambda) \mathbf{y}-F(\mathbf{y})=: v_{\lambda}(\mathbf{y})
$$

have the same stability near $(u, \lambda)=\left(0, \lambda_{0}\right)$. For $\lambda>\lambda_{0}$ with $\lambda-\lambda_{0}$ small, (4.18) admits eight singular points

$$
\pm\left(y_{N}, y_{N+1}\right)=\left(\alpha_{\lambda}, \alpha_{\lambda}\right),\left(\alpha_{\lambda},-\alpha_{\lambda}\right),\left(\gamma_{\lambda}, 0\right),\left(0, \gamma_{\lambda}\right)
$$

where

We note that

$$
\alpha_{\lambda}=\frac{2}{3} \sqrt{\beta}, \quad \gamma_{\lambda}=\sqrt{\frac{4 \beta}{3}}
$$

$$
D v_{\lambda}(\lambda)=\left(\begin{array}{cc}
\beta(\lambda)-\frac{3}{4}\left(3 y_{N}^{2}+2 y_{N+1}^{2}\right) & -3 y_{N} y_{N+1} \\
-3 y_{N} y_{N+1} & \beta(\lambda)-\frac{3}{4}\left(2 y_{N}^{2}+3 y_{N+1}^{2}\right)
\end{array}\right) .
$$

If $y_{N}^{2}=y_{N+1}^{2}=\alpha_{\lambda}^{2}$, then

$$
D v_{\lambda}=\left(\begin{array}{cc}
-\frac{2}{3} \beta(\lambda) & -3 y_{N} y_{N+1} \\
-3 y_{N} y_{N+1} & -\frac{2}{3} \beta(\lambda)
\end{array}\right)
$$

such that

$$
\operatorname{det}\left(D v_{\lambda}-\sigma I\right)=\left(\sigma+\frac{2}{3} \beta\right)^{2}-9 y_{N}^{2} y_{N+1}^{2}=\left(\sigma-\frac{2}{3} \beta\right)(\sigma+2 \beta) .
$$

This implies that $D v_{\lambda}$ has eigenvalues $\frac{2}{3} \beta(\lambda),-2 \beta(\lambda)$. Hence, they are nondegenerate solutions of (4.18) and correspond to saddle points of (4.16). Moreover, these singular solutions corresponds to the steady state solutions $u_{1}^{ \pm}$and $u_{2}^{ \pm}$of (1.1).

On the other hand, if $y_{N}^{2}=\gamma_{\lambda}^{2}$ and $y_{N+1}=0$, then

$$
D v_{\lambda}=\left(\begin{array}{cc}
-2 \beta(\lambda) & 0 \\
0 & -\beta(\lambda)
\end{array}\right)
$$


has eigenvalues $-\beta,-2 \beta$. Thus, $\left(y_{N}, y_{N+1}\right)= \pm\left(\gamma_{\lambda}, 0\right)$ are nondegenerate solutions of (4.18) and correspond to stable nodes of (4.16). Similarly, $\left(y_{N}, y_{N+1}\right)=$ $\pm\left(0, \gamma_{\lambda}\right)$ are regular solutions of (4.18) and correspond to stable nodes of (4.16). It is obvious that these singular solutions corresponds to the steady state solutions $u_{3}^{ \pm}$and $u_{4}^{ \pm}$of (1.1).

The following Figure 1 depicts the center manifold for $\lambda>\lambda_{0}$ with $\lambda-\lambda_{0}$ small.

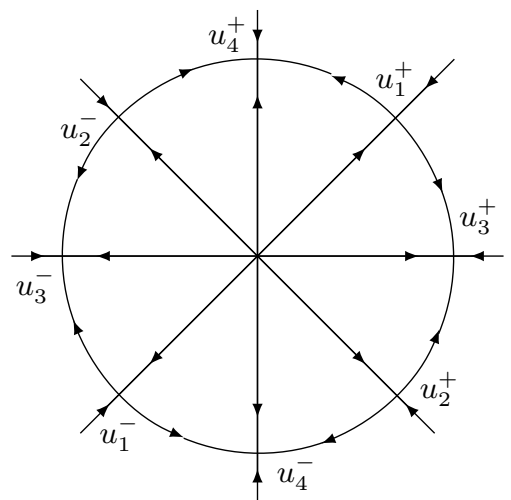

Figure 1.

\section{Periodic case}

In this section, we study the dynamic bifurcation of the SHE with the periodic condition (1.2). Under the periodic boundary condition (1.2), $\mathcal{L}_{\lambda}$ has an eigenvalue sequence on $H$

$$
\beta_{2 n-1}=\beta_{2 n}(\lambda)=\lambda-\lambda_{n}, \quad \lambda_{n}=\left[1-\left(\frac{n \pi}{L}\right)^{2}\right]^{2}, \quad n=1,2, \ldots
$$

with the corresponding eigenvectors

$$
\psi_{2 n-1}(x)=\sin \left(\frac{n \pi x}{L}\right), \quad \psi_{2 n}(x)=\cos \left(\frac{n \pi x}{L}\right) .
$$

Theorem 5.1. Suppose that (2.3) holds true for some $N \in \mathbb{N}$. Then, for the SHE (1.1) defined in $H$, the primary instability of the system happens at $\lambda_{0}=\lambda_{N}=\lambda_{N+1}$. Moreover,

(a) If $\lambda \leq \lambda_{0}$, then $u=0$ is a globally asymptotically stable equilibrium point of (1.1).

(b) For $\lambda>\lambda_{0}$, (1.1) bifurcates from $(u, \lambda)=\left(0, \lambda_{0}\right)$ to an attractor $\mathcal{A}_{\lambda}$ which is homeomorphic to $S^{3}$.

(c) For any bounded open set $U \subset H$ with $0 \in U$, there exists $\varepsilon>0$ such that as $\lambda_{0}<\lambda<\lambda_{0}+\varepsilon, \mathcal{A}_{\lambda}$ attracts $U \backslash \Gamma$ in $H$, where $\Gamma$ is the stable manifold of $u=0$ with codimension 4 .

(d) The bifurcated attractor $\mathcal{A}_{\lambda}$ contains 2 tori and 4 circles which consist of singular points. 
DYNAMIC BIFURCATION OF THE PERIODIC SWIFT-HOHENBERG EQUATION 935

Proof. It comes from (2.3) that

$$
\beta_{n}(\lambda) \begin{cases}<0, & \text { if } \lambda<\lambda_{0} \\ =0, & \text { if } \lambda=\lambda_{0} \\ >0, & \text { if } \lambda>\lambda_{0}\end{cases}
$$

for $n \in \Lambda_{N}=\{2 N-1,2 N, 2 N+1,2 N+2\}$, and $\operatorname{Re} \beta_{n}\left(\lambda_{0}\right)<0$ for all $n \notin \Lambda_{N}$. Hence, the multiplicity of the first eigenvalue is four. Then, proceeding as in the proof of Theorem 4.1, we obtain bifurcated attractors $\mathcal{A}_{\lambda}$ as $\lambda$ crosses $\lambda_{0}$ satisfying the statements (a)-(c).

We prove the statement (d). Let $E_{1}=\operatorname{span}\left\{\psi_{n}: n \in \Lambda_{N}\right\}$ and $E_{2}=E_{1}^{\perp}$ in $H$. Let $P_{j}: H \rightarrow E_{j}$ be the canonical projections and $\mathcal{L}_{j}^{\lambda}=\left.\mathcal{L}_{\lambda}\right|_{E_{j}}$ for $j=1,2$. For simplicity, let $\phi_{i}=\psi_{2 N-2+i}$ for $i=1,2,3,4$ and $\mathbf{y}=\left(y_{1}, y_{2}, y_{3}, y_{4}\right)$. Then, for $u \in H$, we can write $u=\sum_{i=1}^{4} y_{i} \phi_{i}+\Psi(\mathbf{y}, \lambda)$, where $\Psi: E_{1} \rightarrow E_{2}$ is the center manifold function. Proceeding as in the proof of Theorem 4.1, we see that the reduced equations on the center manifold are given by

$$
\frac{d y_{i}}{d t}=\beta(\lambda) y_{i}-g_{i}(\mathbf{y}, \lambda)+o\left(|\mathbf{y}|^{3}\right)
$$

for $i=1,2,3,4$. Here,

$$
g_{i}(\mathbf{y}, \lambda)=\frac{1}{L}\left\langle G\left(y_{1} \phi_{1}+y_{2} \phi_{2}+y_{3} \phi_{3}+y_{4} \phi_{4}\right), \phi_{i}\right\rangle .
$$

We note that for distinct $i, j, k \in\{1,2,3,4\}$,

$$
\begin{array}{ll}
\int_{-L}^{L} \phi_{i}^{4} d x=\frac{3}{4} L, & \int_{-L}^{L} \phi_{i}^{3} \phi_{j} d x=0 \\
\int_{-L}^{L} \phi_{i}^{2} \phi_{j} \phi_{k} d x=0, & \int_{-L}^{L} \phi_{1} \phi_{2} \phi_{3} \phi_{4} d x=0 \\
\int_{-L}^{L} \phi_{1}^{2} \phi_{2}^{2}=\frac{1}{4} L, & \int_{-L}^{L} \phi_{3}^{2} \phi_{4}^{2}=\frac{1}{4} L, \\
\int_{-L}^{L} \phi_{i}^{2} \phi_{j}^{2}=\frac{1}{2} L & \text { for other cases. }
\end{array}
$$

Using these formula, we obtain that

$$
\begin{aligned}
g_{1}(\mathbf{y}, \lambda) & =\frac{3}{4} y_{1}\left(y_{1}^{2}+y_{2}^{2}+2 y_{3}^{2}+2 y_{4}^{2}\right), \\
g_{2}(\mathbf{y}, \lambda) & =\frac{3}{4} y_{2}\left(y_{1}^{2}+y_{2}^{2}+2 y_{3}^{2}+2 y_{4}^{2}\right), \\
g_{3}(\mathbf{y}, \lambda) & =\frac{3}{4} y_{3}\left(2 y_{1}^{2}+2 y_{2}^{2}+y_{3}^{2}+y_{4}^{2}\right), \\
g_{4}(\mathbf{y}, \lambda) & =\frac{3}{4} y_{4}\left(2 y_{1}^{2}+2 y_{2}^{2}+y_{3}^{2}+y_{4}^{2}\right) .
\end{aligned}
$$

If we set $F=\left(g_{1}, g_{2}, g_{3}, g_{4}\right)$, then we have the inequality (4.17) such that the bifurcated attractor $\mathcal{A}_{\lambda}$ is homeomorphic to $S^{3}$ by Theorem 3.3. 
Since the subspace of odd periodic functions is invariant under $\mathcal{L}_{\lambda}+G$ in $H$, the system (5.20) in $\tilde{H}$ is equal to (4.16). This implies that we have singular points of (5.20) given by (4.19). Since SHE is invariant in $H$ under the spacial translation, the steady state solution $\alpha_{\lambda} \phi_{1}+\alpha_{\lambda} \phi_{3}+o\left(\sqrt{\lambda-\lambda_{0}}\right) \in \tilde{H}$ generates singular points in $H$ of (5.20): for $\theta_{1}, \theta_{2} \in \mathbb{R}$,

$$
\begin{aligned}
& \alpha_{\lambda} \sin \left(\frac{N \pi}{L}\left(x+\theta_{1}\right)\right)+\alpha_{\lambda} \sin \left(\frac{(N+1) \pi}{L}\left(x+\theta_{2}\right)\right)+o\left(\sqrt{\lambda-\lambda_{0}}\right) \\
= & \alpha_{\lambda} \cos \frac{N \pi \theta_{1}}{L} \cdot \sin \frac{N \pi x}{L}+\alpha_{\lambda} \sin \frac{N \pi \theta_{1}}{L} \cdot \cos \frac{N \pi x}{L}+ \\
& \alpha_{\lambda} \cos \frac{(N+1) \pi \theta_{2}}{L} \cdot \sin \frac{(N+1) \pi x}{L}+\alpha_{\lambda} \sin \frac{(N+1) \pi \theta_{2}}{L} \cdot \cos \frac{(N+1) \pi x}{L} \\
& \quad+o\left(\sqrt{\lambda-\lambda_{0}}\right) \\
= & y_{1} \phi_{1}+y_{2} \phi_{2}+y_{3} \phi_{3}+y_{4} \phi_{4}+o\left(\sqrt{\lambda-\lambda_{0}}\right) .
\end{aligned}
$$

We note that

$$
y_{1}^{2}+y_{2}^{2}=y_{3}^{2}+y_{4}^{2}=\alpha_{\lambda}^{2} .
$$

This gives a set of singular points in $H$ defined by a two-dimensional torus

$$
\left\{u=y_{1} \phi_{1}+y_{2} \phi_{2}+y_{3} \phi_{3}+y_{4} \phi_{4}+o\left(\sqrt{\lambda-\lambda_{0}}\right): y_{1}^{2}+y_{2}^{2}=y_{3}^{2}+y_{4}^{2}=\alpha_{\lambda}^{2}\right\} \text {. }
$$

A similar argument can be shown to prove the existence of invariant circles. The steady state solution $\left(\gamma_{\lambda}, 0\right)$ in (4.19) generate the following circles in $H$ : for $\theta \in \mathbb{R}$,

$$
\begin{aligned}
& \gamma_{\lambda} \sin \left(\frac{N \pi}{L}(x+\theta)\right)+o\left(\sqrt{\lambda-\lambda_{0}}\right) \\
= & \gamma_{\lambda} \cos \frac{N \pi \theta_{1}}{L} \cdot \sin \frac{N \pi x}{L}+\gamma_{\lambda} \sin \frac{N \pi \theta_{1}}{L} \cdot \cos \frac{N \pi x}{L}+o\left(\sqrt{\lambda-\lambda_{0}}\right) \\
= & y_{1} \phi_{1}+y_{2} \phi_{2}+o\left(\sqrt{\lambda-\lambda_{0}}\right) .
\end{aligned}
$$

Hence we have a set of singular points in $H$ defined by a circle

$$
\left\{u=y_{1} \phi_{1}+y_{2} \phi_{2}+o\left(\sqrt{\lambda-\lambda_{0}}\right): y_{1}^{2}+y_{2}^{2}=\gamma_{\lambda}^{2}\right\} \text {. }
$$

Similarly, the singular point $\gamma_{\lambda} \phi_{3}+o\left(\sqrt{\lambda-\lambda_{0}}\right) \in \tilde{H}$ given in (4.19) generates the invariant circle in $H$

$$
\left\{u=y_{3} \phi_{3}+y_{4} \phi_{4}+o\left(\sqrt{\lambda-\lambda_{0}}\right): y_{3}^{2}+y_{4}^{2}=\gamma_{\lambda}^{2}\right\}
$$

This completes the proof.

\section{References}

[1] E. Bodenschatz, W. Pesch, and G. R Ahlers, Recent Developments in Rayleigh-Bénard Convections, Annual review of fluid mechanics, Vol. 32, 709-778, Annu. Rev. Fluid Mech., 32, Annual Reviews, Palo Alto, CA, 2000.

[2] M. C. Cross and P. C. Hohenberg, Pattern formation outside of equilibrium, Rev. Mod. Phys. 65 (1993), 851-1112. 
DYNAMIC BIFURCATION OF THE PERIODIC SWIFT-HOHENBERG EQUATION 937

[3] P. G. Daniels, D. Ho, and A. C. Skeldon, Solutions for nonlinear convection in the presence of a lateral boundary, Phys. D 178 (2003), no. 1-2, 83-102.

[4] D. Henry, Geometric Theory of Semilinear Parabolic Equations, Lecture Notes in Mathematics 840, Springer, 1981.

[5] C.-H. Hsia and X. Wang, On a Burgers' type equation, Discrete Contin. Dyn. Syst. Ser. B 6 (2006), no. 5, 1121-1139.

[6] T. Ma, J. Park, and S. Wang, Dynamic bifurcation of the Ginzburg-Landau equation, SIAM J. Appl. Dyn. Syst. 3 (2004), 620-635.

[7] _ Addendum to the paper "Two-dimensional infinite prandtl number convection: structure of bifurcated solutions, J. Nonlinear Sci. 17 (2007), no. 3, 199-220", arXiv:0709.1740v1.

[8] T. Ma and S. Wang, Bifurcation Theory and Applications, World Scientific, 2005.

[9] Stability and Bifurcation of Nonlinear Evolution Equations, Science Press, 2007.

[10] — Rayleigh-Bénard convection: dynamics and structure in the physical space, Commun. Math. Sci. 5 (2007), no. 3, 553-574.

[11] — Cahn-Hilliard equations and phase transition dynamics for binary systems, Discrete Contin. Dyn. Syst. Ser. B 11 (2009), 741-784.

[12] L. A. Peletier and V. Rottschäfer, Pattern selection of solutions of the Swift-Hohenberg equation, Phys. D 194 (2004), no. 1-2, 95-126.

[13] L. A. Peletier and J. F. Williams, Some Canonical Bifurcations in the Swift-Hohenberg Equation, SIAM J. Appl. Dyn. Syst. 6 (2007), no. 1, 208-235.

[14] J. Swift and P. C. Hohenberg, Hydrodynamic fluctuations at the convective instability, Phys. Rev. A 15 (1977), 319-328.

[15] A.-M. Wazwaz, New solitary wave solutions to the Kuramoto-Sivashinsky and the Kawahara equations, Appl. Math. Comput. 182 (2006), no. 2, 1642-1650.

[16] M. Yari, Attractor bifurcation and final patterns of the $n$-dimensional and generalized Swift-Hohenberg equations, Discrete Contin. Dyn. Syst. Ser. B 7 (2007), no. 2, 441-456.

JONGMIN HAN

DePARTMENT OF MATHEMATiCS

KYUNG HeE UNIVERSITY

SEOUl 130-701, Korea

E-mail address: jmhan@khu.ac.kr

MASOUD YARI

SCHOOL OF MATHEMATiCS

Institute for Research in Fundamental Sciences (IPM)

P.O.Box 19395-5746, TEHRAN, Iran

E-mail address: myari@indiana.edu, yari@ipm.ir 